\title{
The role of the nurse in the care and management of patients with atopic dermatitis
}

\author{
Harmieke van Os-Medendorp ${ }^{1 *} \mathbb{D}$, Elfie Deprez ${ }^{2} \mathbb{D}$, Nele Maes ${ }^{2}$, Sheila Ryan ${ }^{3} \mathbb{D}$, Karina Jackson $^{4} \mathbb{D}$, \\ Tonya Winders ${ }^{5}$ (D) Linda De Raeve ${ }^{6}$, Christa De Cuyper ${ }^{7,8}$ (D) and Steven Ersser ${ }^{9}$ (D)
}

\begin{abstract}
Background: The purpose of this paper is to provide an overview of key aspects of specialised dermatology nursing practice in the management of patients with moderate to severe atopic dermatitis. The role of dermatology nurse specialists in supporting patients and promoting disease understanding, education and treatment adherence continues to evolve. As features of specialised nursing care can also inform other nursing staff in a wide range of care settings, an overview of key components is examined. Observations presented are from a pan-European perspective and represent the collected view of a group of dermatology nurse specialists, dermatologists and patient advocates following two round-table discussions.

Main body: Atopic dermatitis is a common, chronic, inflammatory disease characterised by erythematous/scaling skin lesions, with often intense pruritus. Disease course is cyclic with periodic disease flares of varying intensity, presenting management challenges to patients and families. Dermatology nurse specialists play a key role in providing education and substantial patient support to improve treatment outcomes and quality of life to patients and their family, delivered within a multidisciplinary team framework. Nurse-led education and 'eczema schools' are of benefit in reducing disease severity and improving quality of life by enhancing self-management, adherence and patient engagement. eHealth tools, such as patient portals or online training platforms, can provide online learning, individualised education, and help to improve engagement. These and other initiatives, such as written action plans, are all essential to improve or maintain treatment adherence, self-management and quality of life.
\end{abstract}

Conclusions: Dermatology nurse specialists play a central role in the assessment and management of moderate to severe atopic dermatitis patients and families. This places them in an ideal position to build strong and often longterm relationships with patients and parents. Such engagement promotes trust, assists in setting realistic expectations of treatment and outcomes, and enhances self-management and engagement in their own care. Providing emotional support, as well as formal and systematic education (including individualised practical advice) all contribute to improved treatment adherence and can enhance the quality of life of patients and their families throughout the course of this long-term condition.

Keywords: Atopic dermatitis, Eczema, Treatment, Holistic care, Nurse specialist, Self-management, Education, Ehealth

\footnotetext{
* Correspondence: harmiekevanos@gmail.com

${ }^{1}$ Saxion, School of Health, Deventer, Enschede, The Netherlands

Full list of author information is available at the end of the article
}

(c) The Author(s). 2020 Open Access This article is licensed under a Creative Commons Attribution 4.0 International License, which permits use, sharing, adaptation, distribution and reproduction in any medium or format, as long as you give appropriate credit to the original author(s) and the source, provide a link to the Creative Commons licence, and indicate if changes were made. The images or other third party material in this article are included in the article's Creative Commons licence, unless indicated otherwise in a credit line to the material. If material is not included in the article's Creative Commons licence and your intended use is not permitted by statutory regulation or exceeds the permitted use, you will need to obtain permission directly from the copyright holder. To view a copy of this licence, visit http://creativecommons.org/licenses/by/4.0/ The Creative Commons Public Domain Dedication waiver (http://creativecommons.org/publicdomain/zero/1.0/) applies to the data made available in this article, unless otherwise stated in a credit line to the data. 


\section{Background}

The purpose of this article is to provide a contemporary overview of the key aspects of specialised dermatology nursing practice in patients with atopic dermatitis (AD) - also known as atopic eczema - and in particular to examine the evolving role of dermatology nurse specialists in supporting patients and promoting disease understanding, education and treatment adherence in those living with moderate to severe $\mathrm{AD}$. A range of different nurse roles exist that may be involved in the care of $\mathrm{AD}$ patients, including general nurses working within dermatology, registered dermatology nurses, and nurses with additional training and responsibilities such as dermatology clinical nurse specialists, advanced nurse practitioners and nurse consultants. For simplicity in this manuscript we have used the term 'dermatology nurse specialists' to refer to those nurses with additional expertise in specialised dermatology nursing care. These observations are from a pan-European perspective formed from a group of specialist dermatology nurses (ED, NM, SR, KJ, SE), a dermatology nurse researcher ( $\mathrm{HvO})$, dermatologists (LDR, CDC) and a patient advocate (TW), who met to develop a consensus on selected topics of specialised dermatology nurse care in AD.

$\mathrm{AD}$ is a chronic, inflammatory skin disease characterised by erythematous and scaling lesions, often with intense pruritus [1]. Disease pathophysiology is complex, involving the interplay of genetic, environmental, and immunological factors. Defects in the epidermal barrier, often associated with gene mutations and polymorphisms in filaggrin and altered lipid metabolism, contribute to epidermal dehydration. This predisposes to immune responses to allergens, skin irritants and microbial proteins with subsequent acute and then chronic cutaneous inflammation. AD is common, affecting $20-30 \%$ of children, with most patients developing symptoms in infancy or early childhood. While most children will have spontaneous remission, persistence into adulthood is seen in approximately $30 \%$ of cases. The overall prevalence of $\mathrm{AD}$ in adults is between 2 and $8 \%$ and although adult-onset $\mathrm{AD}$ is well recognised, most have had symptoms since childhood [2-5]. In $80 \%$ of patients, an atopic background exists ("extrinsic/allergic AD") with the remainder being non-allergic individuals (i.e., those with "intrinsic AD") [6]. Patients with extrinsic AD are at higher risk of contracting other atopic diseases (allergic asthma, allergic rhino-conjunctivitis, and food allergy). Indeed, childhood onset $\mathrm{AD}$ is often a forerunner to the development of one or more atopic conditions in later life - the so called "atopic march" [7]. Other comorbidities are also more common in $\mathrm{AD}$ patients. These include cardiovascular diseases, autoimmune diseases, low mood and issues with self-esteem [8, 9]. Psychological upset and sleep disturbance associated with skin irritation and pruritus have a substantial impact on quality of life (QoL) [10-13].

Typically, the disease course is cyclic with periodic exacerbations/disease flares of varying intensity [2]. The chronic relapsing disease pattern presents challenges for management, which involves ongoing treatment and monitoring for therapeutic response and tolerability. Treatment aims are to alleviate disease symptoms and reduce the frequency and severity of disease flares/exacerbations, to enhance QoL $[14,15]$. A detailed summary of contemporary management approaches is beyond the scope of the present paper; for further detail on diagnosis and treatment, readers are encouraged to review key clinical guidelines and recommendations [3, 4, 14-17] and recent informative reviews $[1,2,18]$. Presented here are the most salient aspects which guide the role of specialised dermatology nursing care in the effective support of patients with AD.

\section{Main text}

\section{Role of the nurse in the patient-centered care team}

$\mathrm{AD}$ disease management may be delivered in different health settings (primary care, day-clinics, and secondary/ tertiary care). The majority of patients have mild disease and are often managed in the primary care/community setting; only up to $10 \%$ are considered to have severe disease [14]. Specialist-led care is primarily delivered on an out-patient or day-clinic basis. Hospital admission for treatment is less common, although it may be provided for patients with severe or recalcitrant disease. In cases where a structured treatment and education program is considered necessary, in-patient care can help optimise topical therapy with out-patient follow-up, thereby reducing the need for systemic immunosuppressive or biologic therapy [16].

Nurses in all settings play a vital and valued role in providing care for the patient with $\mathrm{AD}[17,18]$ (Fig. 1). Involvement of dermatology nurse specialists in providing and supporting clinical care allows the dermatologist to dedicate greater time to clinical aspects of patient consultations. The nurse - patient consultation addresses a range of aspects such as disease assessment, structured education and emotional support. However, no standardised format for consultations currently exists, nor consensus on what key elements should be included. On an individual patient basis, this may be informed by observations derived from multidisciplinary team discussions. A standardised nurse consultation 'tool' could be valuable as a framework to ensure that all appropriate relevant items are addressed and could allow a more consistent approach to monitoring disease course and patient support.

Ideally, moderate to severe AD care is delivered within a multidisciplinary team, with the focus on delivering 


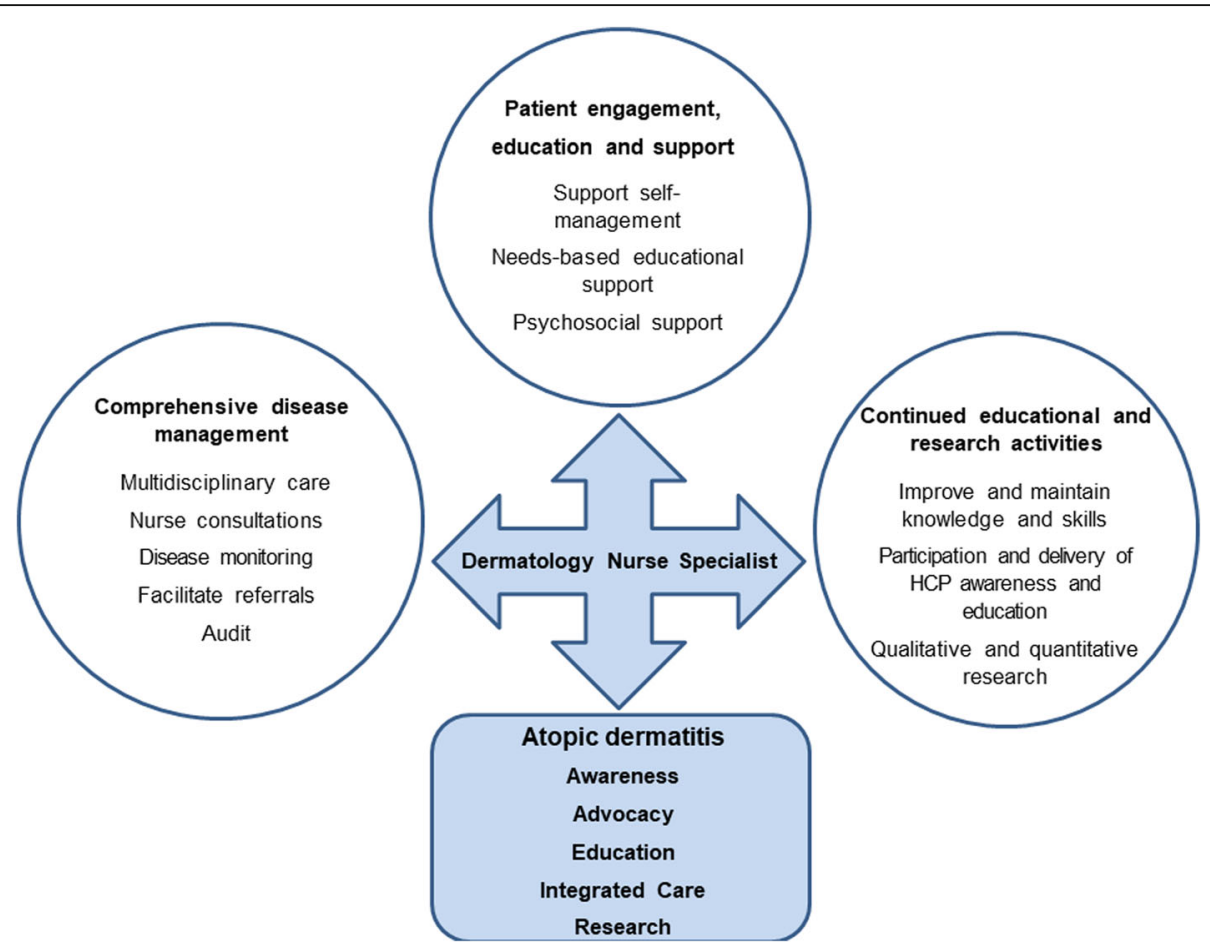

Fig. 1 Overview of the role of dermatology nurse specialist in atopic dermatitis care

person-centered care. In this integrated approach, communication, education, and treatment need to be individualised to suit the patient's needs within a shared decision-making process. This must take into consideration personal disease history, severity and comorbidities, and match realistic care expectations and personal preferences to the best scientific evidence available. Shared decision making can be supported by patient decision aids [19].

While the composition of the team varies between settings, institutions and across countries, dermatologists, dermatology nurse specialists, other nursing staff, pharmacists, along with psychologist or psychiatrist support, dieticians and other staff, all play a role [20]. Multidisciplinary initiatives and interventions, including education about the condition and its management, as well as psychological and behavioural programmes, may have a positive effect on outcomes in moderate to severe $\mathrm{AD}$ [21, 22].

Within such a team, nurses often serve as the principal contact between the patient (and their family) and the lead physician and other team members to support continuity of care [23]. Nurses tend to have well-established patient rapport and empathy skills, especially when addressing practical problems in eczema management, and are highly valued by patients [24]. They are, therefore ideally placed for continuity of primary patient contact at every stage of care provision, to both develop and document the patient's clinical profile at the initial consultation and engage with patients through education and training throughout their care. In addition, dermatology nurse specialists may provide education and training to fellow healthcare workers, both within the team and in other settings, such as in primary care. Many are also actively involved in clinical research, integrated with clinical care.

Establishing and managing realistic patient expectations in their treatment and their role in selfmanagement is fundamental. These may vary greatly in patients (and the parents of children) as may engagement in the shared decision-making process. Some parents/patients are highly motivated and informed, while others are more reluctant or cautious when seeking information and receiving appropriate clinical management; some may rely completely upon guidance from the healthcare team. Initial attitudes may change during the clinical disease course, and with patient experience and age. Providing patients/parents with the most appropriate and realistic information on their disease status, including the efficacy, safety, and suitability of proposed treatments, based upon their individual needs at each stage of the disease, is vital. Such information should be delivered in a clear, consistent fashion by all members of the healthcare team to inspire confidence and minimise 
confusion or misunderstanding on the part of the patient or parent [14, 15, 25-28].

Active engagement of the patient in decision making is paramount. A genuine sense of personal responsibility should be encouraged - especially regarding the importance of treatment adherence in achieving optimal self-management and optimal outcomes [19]. For example, while skincare and application of topical therapies is time consuming, and patient dissatisfaction contributes to poor treatment adherence, this may be overcome with support and education. Even with good understanding and acceptance of the chronic relapsing nature of moderate to severe $\mathrm{AD}$, patients will naturally experience disappointment during adverse changes in their condition and nurses can offer reassurance and hope. Nurses can guide patients adapt through the disease cycle and therapy changes. Patients should be informed and understand that treatment escalation may be an effective and necessary strategy that is often only needed as a temporary measure to control disease exacerbations. Correct management of the medication, including frequency of administration and adaptation of the potency of the drugs/ointments, is crucial. Greater understanding will improve the patient's adherence and therefore, enhance the clinical outcome.

\section{Overview of clinical management}

Diagnosis of AD is based on family and personal history and clinical features. Most diagnostic criteria consider cutaneous aspects (including dry skin \pm erythema, induration, and scaling), pruritus and a personal or family history of allergic skin or respiratory disease (i.e., $\mathrm{AD}$, asthma or allergic rhinoconjunctivitis) as major diagnostic criteria, with disease chronicity and/or a relapsing pattern, and early onset $(<2$ years of age) supportive of a diagnosis [29, 30]. Identification of additional trigger factors (food, environmental and job-related factors) is crucial in disease management. Establishing a complete profile of the patient is indispensable to determine the therapeutic strategy. Dermatology nurse specialists are often involved in skilled clinical assessment, gathering data to inform the diagnostic process and the estimation of disease severity and QoL impact.

Most treatment recommendations are based on disease severity and therapeutic response; a framework for categorizing $\mathrm{AD}$ severity as mild, moderate, or severe is important to understand and anticipate an individual's treatment needs $[14,15,25]$. While treatment decisions can be complex, in part due to the wide variety of topical and systemic therapies available, most evidence-based clinical practice guidelines from specialist bodies in Europe and the US provide treatment recommendations on the basis of disease severity and treatment response [14, 15, 25-28].

Since AD is characterised by exacerbation and disease flares, followed by remission, severity should be assessed both at presentation and throughout the disease course; sequential measurements are ideal to generate reliable estimates [26]. A wide range of validated disease scoring tools are available, such as the Scoring Atopic Dermatitis Index (SCORAD) - with clinical and patient-oriented versions (PO-SCORAD), each assessing the extent and severity of skin involvement and also a subjective assessment of the severity of pruritus and sleep disturbance [31-33]. The Eczema Area and Severity Index (EASI) assesses the extent and severity of skin disease only [32]. Patient-reported outcome measures (PROMs) are also used for assessing symptoms or impact on QoL. These may be disease-specific - such as the validated PatientOriented Eczema Measure (POEM) and more generic dermatology-focused tools such as the Dermatology Life Quality Index (DLQI) [34-36]. Although these tools may not always be employed in the clinical practice setting, their use is on the increase and, by using them, nurses can contribute to the objective assessment to inform disease management and decisions about treatment and its evaluation. Discussing these measures with patients is often useful to provide context and help them gain a better understanding of their disease and its impact.

\section{Treatment aspects}

The principles of treatment for moderate to severe $\mathrm{AD}$ are four-fold; (1) identification and avoidance of trigger factors; (2) skin rehydration to repair/restore the impaired skin barrier; (3) reducing itching/pruritus; (4) reducing skin inflammation, especially during disease flares, via topical and systemic therapies. Dermatology nurse specialists provide a crucial resource for patient education and support in all aspects of treatment.

Environmental irritants and known allergens can aggravate affected skin of AD patients and promote disease flares [14]. Well-recognised irritants include fabrics such as acrylic or wool, household chemicals (bleach or solvents), and fragrances. Daily use of emollients to reduce moisture loss is the mainstay of treatment. This has been shown to reduce pruritus and may also have a steroidsparing effect, reducing the amount of topical corticosteroids (TCS) required [14, 37-39]. Specific skincare products such as emollients should be recommended, and according to personal needs lotions, creams or ointments that are preferred by the patient. Most over-thecounter products seem to be as effective as specialist prescription formulations [14]. Dermatology nurse specialists trained in $\mathrm{AD}$ management have a good knowledge of available products and are skilled in providing 
advice to patients who have difficulty in finding an emollient regime that suits their individual needs and preferences.

Key factors determining emollient use are the level of dryness of the skin and patient preference (i.e., one they are most likely to use). As individual preference is important to promote adherence [40]; it may be necessary to trial a range of products to establish a preferred combination of products. In principle, emollients should be applied directly on the skin after bathing and then applied on 2-3 further occasions throughout the day. Identifying the appropriate skincare regimen may take time, and once established, its use in everyday life may be time-consuming, and these aspects may be a source of patient or parental concern.

Wet-wrap therapy is a useful adjunct to standard skincare and topical therapies [41-44]. Their clinical value has been highlighted in Nicol's robust large nurse-led study [41], although recent systematic review evidence calls for further research [42]. Wet wrapping involves the application of emollients or TCS, which are then covered by a layer of wet bandages or clothing and then by a further layer of dry clothing. Wet-wraps are especially useful in children as they provide a barrier to scratching affected skin in acute flares (although they are contraindicated when eczema is infected) [41-44]. A principal drawback is poor adherence, chiefly due to inadequate patient education. Nurse support in wet-wrap technique through demonstration is especially helpful, and particularly so for children, where engagement of both parent and child can be enhanced by incorporation of wet-wrapping into their lifestyle. Play strategies, such as "reward stickers" or wetwrapping a favourite doll, can increase the acceptability of this technique to the child.

Medical therapies include topical agents, in particular TCS or topical calcineurin inhibitors (TCIs), phototherapy and systemic or biologic agents (immunosuppressants or biologic therapies e.g. dupilumab). Evidencebased guidelines recommend a stepwise approach to agent selection with a range of topical agents being considered as first-line before systemic immunosuppressants or biologic therapies are considered $[14,15,25-28]$ (Fig. 2).

In general, the therapeutic approach is initial use of medium potency or higher-dose TCS preparations prior to TCIs. In severe AD, initial use of higher potency TCS may be preferred. When used appropriately TCS are safe; local side effects with longer-term use are uncommon and systemic side effects are rare. Nevertheless, safety may be an important aspect of patients often undue concerns or overt 'steroid phobia', especially in parents of affected children, which contributes to reduced treatment adherence. Explanation of the benefits and risks are, therefore, key aspects of patient education and reassurance $[45,46]$.

Dermatology nurse specialists are actively involved in delivering phototherapy to patients using established protocols, although treatment may be time-consuming for patients (and their families). Phototherapy is usually given in schedules of 3-5 weekly treatments for 6-12 weeks, with discontinuation if little response is seen within 2 months [14]. Treatment with existing care

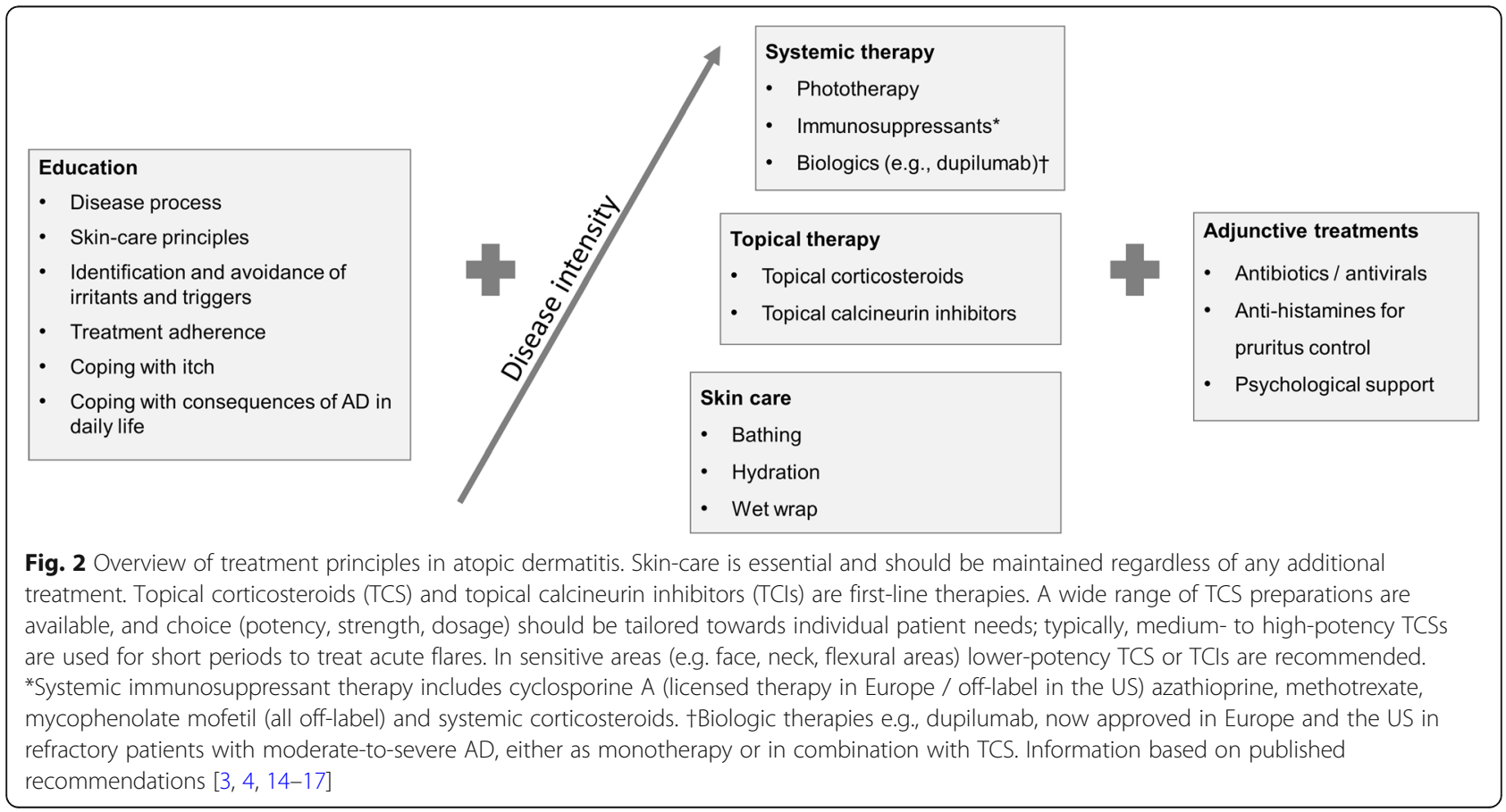


(emollients \pm TCS) should be continued to minimise risk of acute flares. However, access to established centres may limit the utility of phototherapy to patients with moderate to severe $\mathrm{AD}$ who may benefit [14].

Patients with moderate-to severe AD may require systemic therapy with immunosuppressants (cyclosporine A, azathioprine, methotrexate, and mycophenolate mofetil) or targeted biologics such as dupilumab. Whilst decisionmaking should be shared between the patient and the physician to take account of patient preference, screening for the suitability of immunosuppressants or biologics, along with monitoring for adverse effects and clinical responses, are often coordinated by dermatology nurse specialists [15]. This may involve the ordering and review of various blood investigations according to local guidelines and protocols, and dermatology nurse specialists are increasingly involved in monitoring patients on therapies. When stepping-up treatment to systemic or biologic therapies, comprehensive discussions with patients or their family may be required to explain the rationale for such treatment and how duration of systemic treatment would be guided by clinical response and tolerability. Education on the more common side-effects of immunosuppressants or biologics and to provide perspective on the risk-benefit profile of specific agents is also important to raise awareness and reassure patients and their family. In addition, many of these agents may be given as subcutaneous injection (azathioprine, methotrexate, dupilumab) and while in some circumstances nurse-administration will be preferred, self-administration is a more practical option. As such, education and training in self-injection is essential.

\section{Promoting wellbeing}

Patients with moderate to severe $\mathrm{AD}$ are at an increased risk of a wide range of co-morbidities, including cardiovascular, autoimmune, and psychiatric diseases $[8,9]$. The systemic impact of $\mathrm{AD}$ is such that it has been considered by many - similar to psoriasis - to be a systemic disease [47]. An essential aspect of holistic, integrated care is the promotion of healthier lifestyles and supporting adherence with recommended medical care. This is important at all ages. For example, children with $\mathrm{AD}$ should be vaccinated in line with local or national vaccination schedules, although where possible, vaccines should be avoided during acute flares [14]. Healthy lifestyle changes may be required for some adult $\mathrm{AD}$ patients with moderate to severe disease $[8$, 9]. When appropriate, efforts to reduce alcohol use (and tobacco cessation) and promote weight-loss, with development of a regular exercise regime may all be important aspects of holistic patient care to improve wellbeing [9].

Other important aspects of wellbeing include the identification of those patients with a psychological disturbance. It should be recognised that adverse mood and poorer QoL can also develop in families of patients with moderate to severe AD. These may be formally measured and monitored by appropriate validated measures (e.g., Hospital Anxiety and Depression Scale) [48], although other factors beyond overt depression can be a particular burden. An important judgement is the need to assess when to instigate specialist referral to psychiatric or clinical psychology services.

Although beyond the scope of this article, a range of factors may adversely impact the patient's sense of wellbeing. Sleep disturbance, self-esteem and social anxiety, intimate personal relationships and financial concerns due to treatment costs and impact upon work productivity and absences, can each contribute to lower mood and poorer disease-related life quality. Anxiety frequently relates to poor adherence to therapies, which may itself be associated with factors such as steroid aversion [49]. Nurses are well placed to provide counselling to patients and parents, actively listen; they may also use motivational interviewing to help minimise these impacts and support better adherence.

\section{Patient education}

Nurses play a key role in the development and provision of systematic education on $\mathrm{AD}$, both to patients, their relatives, and the wider healthcare community, on disease background, standards of care, and detailing risks and benefits of the available recommended treatments. In most therapeutic patient education (TPE) programmes, nurses often play a primary role in providing evidence-based and often individualised information to support self-management and promote active participation in shared decision making [20, 50-54]. Educational needs that are required to support self-care varies between patients, and needs-assessment can be difficult; simple scoring tools have been developed for this purpose [55]. While such education should be universal it is of particular importance in those patients with recalcitrant or refractory disease, or where treatment adherence or treatment concerns exist, and for those patients or families with poorer QoL, or who require additional psychological support [50, 52].

Two main service delivery models for education delivery exist; nurse-led focused group education and a broader multidisciplinary "eczema school" approach [50, 56]. To date, most experience and evaluation of the benefits of education is in children, where education may be directed towards children and their parents. There is no strong evidence that either approach is more effective as no direct comparative studies have been conducted. A recent Cochrane review of educational interventions in children concluded that either approach may lead to improvements in disease severity and QoL [56], although the eczema school approach is much more resource intensive. The "eczema school approach" was pioneered in 
Europe and has now also been used in the US with some success [57]. Eczema schools provide a structured intervention program for patients or parents of children with AD. Educational components include: disease information, practical advice on skin care and avoidance strategies, and nutritional advice. Psychological and social support are also provided. In the UK, nurse-led education such as the Eczema Education Programme (EEP) involving group education and social learning, has also been well received by parents and preliminary evidence indicates its clinical and QoL benefits [17, 58, 59], although further evaluative trial evidence is required. Although time-consuming, individualised education within an out-patient or day-clinic, may be more appropriate when delivering specific patient training on skincare and the use of topical therapies in adult patients with more complex educational needs.

Patients with moderate to severe AD may suffer a sense of isolation and learning about fellow patients' experiences can help to alleviate this. The DIPEx (personal experiences of health and illness) international collaboration is a useful resource, providing videos of patient interviews on a wide range of medical conditions to provide genuine insight into the experiences of patients, family, and carers [60-62]. While patients should be encouraged to learn about their condition independently, and the internet is a valuable source of information for some patients, - misleading or adverse impressions of appropriate therapy for moderate to severe AD can be made [63]. Nurses can provide guidance on appropriate reliable evidence-based information sources.

\section{eHealth strategies for management}

Electronic digital health services (eHealth) - the use of information and communication technologies for health [64] - provide an important additional avenue to support $\mathrm{AD}$ care $[65,66]$. In addition to assisting in scheduling appointments and prescription reminders, eHealth offers the ability to provide online medical consultations, with different studies reporting equivalent improvements in $\mathrm{AD}$ outcomes and QoL with online follow-up appointments compared with conventional face-to-face consultations [67-69].

eHealth strategies are considered favourably by both healthcare workers and patients, and nurses are an important contact in supporting access to eHealth resources. Such resources can range from static tools (providing core disease information) to more dynamic ones providing interactive online learning, selfmanagement training or a personal ePortal providing patient-specific access to their electronic health records [70]. Mobile apps are available to monitor disease severity or symptoms such as itch [71, 72], and it is likely that the use of such tools by patients will increase.
Providing a suite of tools such as these can deliver individualised education and engagement [73, 74]. Increasingly, such tools are being developed and shared by national bodies or dedicated institutions e.g., those developed by the University Medical Center of Utrecht in the Netherlands or by the Foundation for Atopic Dermatitis [75, 76] In the US, the American College of Allergy, Asthma \& Immunology, in partnership with the Allergy \& Asthma Network patient organization, have developed an interactive tool to support patients with moderate-to-severe AD in shared decision making with their physician during a clinical consultation [77].

The educational and eHealth strategies described above can inform and support adherence. The chronic relapsing disease course, which requires rigorous attention to skincare, and different treatments over a prolonged period, can translate into a complex management plan, with episodes of disappointment and frustration regarding outcomes with topical therapies. This can result in reduced levels of adherence and subsequently poorer outcomes in moderate to severe AD.

The use of written eczema action plans, which provide patients or parents with a checklist of instructions, are also valuable to support self-management. Plans such as these are already an established aspect of asthma care, and although data on their effectiveness in AD is limited, they would seem to provide an important aspect to supporting care in $\mathrm{AD}$ and patient engagement [78-80]. Action plans for $\mathrm{AD}$ have been developed by a number of professional organizations, including the American Academy of Dermatology [81].

\section{Conclusions}

Dermatology nurse specialists are key players in the assessment and management of patients with moderate to severe AD. Their central role within a multidisciplinary team places them in an ideal position to build strong and often long-term relationships with patients and parents. Such engagement promotes trust, assists in setting realistic expectations of treatment and outcomes, and enhances self-management and engagement in their own care. Providing emotional support, as well as formal and systematic education and individualised practical advice, may contributes to improved treatment adherence and can enhance QoL of patients and their families throughout the course of this chronic disease. The aspects described in this review may have value in educating nonspecialist nursing staff in the evolving role of dermatology nurse specialists in moderate to severe AD patient management and holistic care.

\section{Abbreviations}

AD: Atopic dermatitis; DLQI: Dermatology Life Quality Index; EASI: Eczema Area and Severity Index; EEP: Eczema Education Programme; PROMs: Patientreported outcome measures; QoL: Quality of life; SCORAD: Scoring Atopic 
Dermatitis Index; TCls: Topical calcineurin inhibitors; TCS: Topical corticosteroids; TPE: Therapeutic patient education

\section{Acknowledgements}

The authors wish to thank Valentina Guasconi (IntraMed Communications, Milan) and lain O'Neill (medical writer) for coordinating the meeting and supporting manuscript development.

\section{Authors' contributions}

TW conceived the review together with panel participants (HvO, ED, NM, SR, LDR, CDC and SE). The manuscript was developed from meeting notes with input from all panel participants (HVO, ED, NM, SR, TW, LDR, CDC and SE). KJ provided critical review of the paper. All authors reviewed manuscript drafts, provided critical feedback and approved the final manuscript.

\section{Funding}

This manuscript was developed from a preliminary meeting followed by an independent round-table discussion held on 16th September 2018 in Paris, France, organised by the Global Allergy \& Airways Patient Platform (GAAPP) with financial support from Sanofi Genzyme and Regeneron. The sponsors had no role in the topics discussed or reported in this manuscript.

\section{Availability of data and materials}

Not applicable.

\section{Ethics approval and consent to participate}

Not applicable.

\section{Consent for publication}

Not applicable.

\section{Competing interests}

CDC has received payment from GAAPP for coordinating a preparative meeting and honoraria from Novartis for serving as a speaker and as a study investigator (AD studies). HvO has received expense reimbursement from GAAPP for contribution to the meeting and reports grants to her supporting institution (University Medical Center Utrecht) from Sanofi for AD studies. LDR received payment from the GAAPP for lecturing and contributing to the preparative meeting, and received non-financial support from SanofiGenzyme.

All other authors declare that they have no conflict of interest.

\section{Author details}

'Saxion, School of Health, Deventer, Enschede, The Netherlands.

2Department of Dermatology, University Hospital Ghent, Ghent, Belgium.

${ }^{3}$ Department of Dermatology, University Hospital Limerick, Limerick, Ireland. ${ }^{4}$ St John's Institute of Dermatology, Guy's and St Thomas' Foundation NHS Trust, London, UK. ${ }^{5}$ Allergy \& Asthma Network / Global Allergy \& Airways Patient Platform (GAAPP), Vienna, VA, USA. ${ }^{6}$ Department of Dermatology, Universitair Ziekenhuis Brussel (UZ Brussel), Brussels, Belgium. ${ }^{7}$ Department of Dermatology, AZ Sint Jan, Brugge-Oostende, AV, Belgium. ${ }^{8}$ EADV-Nurse Association Working group Coordinator, Lugano, Switzerland. ${ }^{9}$ Department of Nursing Science, Bournemouth University, Poole, UK

Received: 24 April 2019 Accepted: 20 October 2020

Published online: 04 November 2020

\section{References}

1. Kapur S, Watson W, Carr S. Atopic dermatitis. Allergy, Asthma Clin Immunol. 2018;14(Suppl 2):52.

2. Weidinger S, Novak N. Atopic dermatitis. Lancet. 2016;387(10023):1109-22.

3. Drucker AM. Atopic dermatitis: burden of illness, quality of life, and associated complications. Allergy Asthma Proc. 2017:38(1):3-8.

4. Silverberg Jl, Hanifin JM. Adult eczema prevalence and associations with asthma and other health and demographic factors: a US population-based study. J Allergy Clin Immunol. 2013;132(5):1132-8.

5. Deckers IA, McLean S, Linssen S, Mommers M, van Schayck CP, Sheikh A Investigating international time trends in the incidence and prevalence of atopic eczema 1990-2010: a systematic review of epidemiological studies. PLoS One. 2012;7(7):e39803.
6. Tokura Y. Extrinsic and intrinsic types of atopic dermatitis. J Dermatol Sci. 2010;58(1):1-7.

7. Hill DA, Spergel JM. The atopic march: critical evidence and clinical relevance. Ann Allergy Asthma Immunol. 2018;120(2):131-7.

8. Andersen YMF, Egeberg A, Skov L, Thyssen JP. Comorbidities of atopic dermatitis: beyond rhinitis and asthma. Curr Dermatol Rep. 2017;6(1):35-41.

9. Silverberg Jl. Associations between atopic dermatitis and other disorders. F1000Res. 2018:7:303.

10. Sibbald C, Drucker AM. Patient burden of atopic dermatitis. Dermatol Clin 2017;35(3):303-16.

11. Holm JG, Agner T, Clausen ML, Thomsen SF. Quality of life and disease severity in patients with atopic dermatitis. J Eur Acad Dermatol Venereol. 2016:30(10):1760-7.

12. Jeon C, Yan D, Nakamura M, Sekhon S, Bhutani T, Berger T, et al. Frequency and Management of Sleep Disturbance in adults with atopic dermatitis: a systematic review. Dermatol Ther (Heidelb). 2017;7(3):349-64.

13. Chang YS, Chiang BL. Sleep disorders and atopic dermatitis: a 2-way street? J Allergy Clin Immunol. 2018;142(4):1033-40.

14. Wollenberg A, Barbarot S, Bieber T, Christen-Zaech S, Deleuran M, FinkWagner A, et al. Consensus-based European guidelines for treatment of atopic eczema (atopic dermatitis) in adults and children: part I. J Eur Acad Dermatol Venereol. 2018;32(5):657-82.

15. Wollenberg A, Barbarot S, Bieber T, Christen-Zaech S, Deleuran M, FinkWagner A, et al. Consensus-based European guidelines for treatment of atopic eczema (atopic dermatitis) in adults and children: part II. J Eur Acad Dermatol Venereol. 2018;32(6):850-78.

16. van der Schaft J, Keijzer WW, Sanders KJ, de Groot JJ, van den Bersselaar DL, van Os-Medendorp $\mathrm{H}$, et al. Is there an additional value of inpatient treatment for patients with atopic dermatitis? Acta Derm Venereol. 2016; 96(6):797-801.

17. Jackson K, Ersser SJ, Dennis H, Farasat H, More A. The eczema education Programme: intervention development and model feasibility. J Eur Acad Dermatol Venereol. 2014;28(7):949-56

18. Courtenay M, Carey N. Nurse-led care in dermatology: a review of the literature. Br J Dermatol. 2006;154(1):1-6.

19. Tan J, Linos E, Sendelweck MA, van Zuuren EJ, Ersser S, Dellavalle RP, et al. Shared decision making and patient decision aids in dermatology. $\mathrm{Br} J$ Dermatol. 2016:175(5):1045-8.

20. Skypala IJ, de Jong NW, Angier E, Gardner J, Kull I, Ryan D, et al. Promoting and achieving excellence in the delivery of integrated allergy care: the European academy of Allergy \& Clinical Immunology competencies for allied health professionals working in allergy. Clin Transl Allergy. 2018;8:31.

21. LeBovidge JS, Elverson W, Timmons KG, Hawryluk EB, Rea C, Lee M, et al. Multidisciplinary interventions in the management of atopic dermatitis. J Allergy Clin Immunol. 2016;138(2):325-34.

22. Spielman SC, LeBovidge JS, Timmons KG, Schneider LC. A review of multidisciplinary interventions in atopic dermatitis. J Clin Med. 2015:4(5): 1156-70.

23. Lamb A, Martin-Misener R, Bryant-Lukosius D, Latimer M. Describing the leadership capabilities of advanced practice nurses using a qualitative descriptive study. Nurs Open. 2018;5(3):400-13.

24. Munidasa D, Lloyd-Lavery A, Burge S, McPherson T. What should general practice trainees learn about atopic eczema? J Clin Med. 2015:4(2):360-8.

25. Boguniewicz M, Alexis AF, Beck LA, Block J, Eichenfield LF, Fonacier $L$, et al. Expert perspectives on Management of Moderate-to-Severe Atopic Dermatitis: a multidisciplinary consensus addressing current and emerging therapies. J Allergy Clin Immunol Pract. 2017:5(6):1519-31.

26. Simpson EL, Bruin-Weller M, Flohr C, Ardern-Jones MR, Barbarot S, Deleuran $M$, et al. When does atopic dermatitis warrant systemic therapy? Recommendations from an expert panel of the international eczema council. J Am Acad Dermatol. 2017;77(4):623-33.

27. Drucker AM, Eyerich K, de Bruin-Weller MS, Thyssen JP, Spuls PI, Irvine AD, et al. Use of systemic corticosteroids for atopic dermatitis: international eczema council consensus statement. Br J Dermatol. 2018:178(3):768-75.

28. Eichenfield LF, Ahluwalia J, Waldman A, Borok J, Udkoff J, Boguniewicz M. Current guidelines for the evaluation and management of atopic dermatitis: a comparison of the joint task force practice parameter and American Academy of Dermatology guidelines. J Allergy Clin Immunol. 2017;139(4S): S49-57.

29. Hanifin JMRG. Diagnostic features of atopic dermatitis. Acta Derm Venereol. 1980;92(Suppl):44-7. 
30. Williams HC, Burney PG, Hay RJ, Archer CB, Shipley MJ, Hunter JJ, et al. The U.K. working Party's diagnostic criteria for atopic dermatitis. I. Derivation of a minimum set of discriminators for atopic dermatitis. Br J Dermatol. 1994;131(3):383-96.

31. Stalder JF, Barbarot S, Wollenberg A, Holm EA, De Raeve L, Seidenari S, et al. Patient-oriented SCORAD (PO-SCORAD): a new self-assessment scale in atopic dermatitis validated in Europe. Allergy. 2011;66(8):1114-21.

32. Chopra R, Vakharia PP, Sacotte R, Patel N, Immaneni S, White T, et al. Severity strata for eczema area and severity index (EASI), modified EASI, scoring atopic dermatitis (SCORAD), objective SCORAD, atopic dermatitis severity index and body surface area in adolescents and adults with atopic dermatitis. Br J Dermatol. 2017;177(5):1316-21.

33. Silverberg JI, Gelfand JM, Margolis DJ, Fonacier L, Boguniewicz M, Schwartz $L B$, et al. Severity strata for POEM, PO-SCORAD, and DLQI in US adults with atopic dermatitis. Ann Allergy Asthma Immunol. 2018;121(4):464-71.

34. Vakharia PP, Cella D, Silverberg Jl. Patient-reported outcomes and quality of life measures in atopic dermatitis. Clin Dermatol. 2018;36(5):616-30.

35. Vakharia PP, Chopra R, Sacotte R, Patel N, Immaneni S, White T, et al. Severity strata for five patient-reported outcomes in adults with atopic dermatitis. Br J Dermatol. 2018:178(4):925-30.

36. Vakharia PP, Chopra R, Sacotte R, Patel N, Immaneni S, White T, et al. Validation of patient-reported global severity of atopic dermatitis in adults. Allergy. 2018;73(2):451-8

37. Eberlein B, Eicke C, Reinhardt HW, Ring J. Adjuvant treatment of atopic eczema: assessment of an emollient containing $\mathrm{N}$-palmitoylethanolamine (ATOPA study). J Eur Acad Dermatol Venereol. 2008;22(1):73-82.

38. Szczepanowska J, Reich A, Szepietowski JC. Emollients improve treatment results with topical corticosteroids in childhood atopic dermatitis: a randomized comparative study. Pediatr Allergy Immunol. 2008;19(7):614-8.

39. van Zuuren EJ, Fedorowicz Z, Christensen R, Lavrijsen A, Arents BWM. Emollients and moisturisers for eczema. Cochrane Database Syst Rev. 2017; 2:CD012119

40. Santer M, Muller I, Yardley L, Lewis-Jones S, Ersser S, Little P. Parents' and carers' views about emollients for childhood eczema: qualitative interview study. BMJ Open. 2016;6(8):e011887.

41. Nicol NH, Boguniewicz M, Strand M, Klinnert MD. Wet wrap therapy in children with moderate to severe atopic dermatitis in a multidisciplinary treatment program. J Allergy Clin Immunol Pract. 2014;2(4):400-6.

42. Gonzalez-Lopez G, Ceballos-Rodriquez RM, Gonzalez-Lopez JJ, Feito Rodriguez M, Herranz-Pinto P. Efficacy and safety of wet wrap therapy for patients with atopic dermatitis: a systematic review and meta-analysis. $\mathrm{Br} J$ Dermatol. 2017;177(3):688-95.

43. Izadi N, Leung DYM. Clinical approach to the patient with refractory atopic dermatitis. Ann Allergy Asthma Immunol. 2018;120(1):23-33 e21.

44. Nicol NH, Boguniewicz M. Wet wrap therapy in moderate to severe atopic dermatitis. Immunol Allergy Clin N Am. 2017;37(1):123-39.

45. El Hachem M, Gesualdo F, Ricci G, Diociaiuti A, Giraldi L, Ametrano O, et al. Topical corticosteroid phobia in parents of pediatric patients with atopic dermatitis: a multicentre survey. Ital J Pediatr. 2017;43(1):22.

46. Stalder JF, Aubert $H$, Anthoine E, Futamura M, Marcoux D, Morren MA, et al. Topical corticosteroid phobia in atopic dermatitis: international feasibility study of the TOPICOP score. Allergy. 2017;72(11):1713-9.

47. Brunner PM, Silverberg Jl, Guttman-Yassky E, Paller AS, Kabashima K, Amagai $M$, et al. Increasing comorbidities suggest that atopic dermatitis is a systemic disorder. J Invest Dermatol. 2017;137(1):18-25.

48. Zigmond AS, Snaith RP. The hospital anxiety and depression scale. Acta Psychiatr Scand. 1983;67(6):361-70.

49. Li AW, Yin ES, Antaya RJ. Topical corticosteroid phobia in atopic dermatitis: a systematic review. JAMA Dermatol. 2017;153(10):1036-42.

50. Nicol NH, Ersser SJ. The role of the nurse educator in managing atopic dermatitis. Immunol Allergy Clin N Am. 2010;30(3):369-83.

51. Thompson DL, Thompson MJ. Knowledge, instruction and behavioural change: building a framework for effective eczema education in clinical practice. J Adv Nurs. 2014;70(11):2483-94.

52. Barbarot S, Stalder JF. Therapeutic patient education in atopic eczema. $\mathrm{Br} J$ Dermatol. 2014;170(Suppl 1):44-8.

53. Stalder JF, Bernier C, Ball A, De Raeve L, Gieler U, Deleuran M, et al. Therapeutic patient education in atopic dermatitis: worldwide experiences. Pediatr Dermatol. 2013;30(3):329-34.

54. LeBovidge J, Borok J, Udkoff J, Yosipovitch G, Eichenfield LF. Atopic dermatitis: therapeutic care delivery: therapeutic education, shared decisionmaking, and access to care. Semin Cutan Med Surg. 2017;36(3):131-6.
55. Cowdell F, Ersser SJ, Gradwell C, Thomas PW. The person-centered dermatology self-care index: a tool to measure education and support needs of patients with long-term skin conditions. Arch Dermatol. 2012;148(11):1251-5.

56. Ersser SJ, Cowdell F, Latter S, Gardiner E, Flohr C, Thompson AR, et al. Psychological and educational interventions for atopic eczema in children. Cochrane Database Syst Rev. 2014;1:CD004054.

57. Grossman SK, Schut C, Kupfer J, Valdes-Rodriguez R, Gieler U, Yosipovitch G. Experiences with the first eczema school in the United States. Clin Dermatol. 2018:36(5):662-7.

58. Ersser SJ, Farasat H, Jackson K, Dennis H, Sheppard ZA, More A. A service evaluation of the eczema education Programme: an analysis of child, parent and service impact outcomes. Br J Dermatol. 2013;169(3):629-36.

59. Jackson K. The eczema education Programme. An innovation to help families of children with eczema. Allergy Newsletter. 2016;118:29-37.

60. Ziebland S, Herxheimer A. How patients' experiences contribute to decision making: illustrations from DIPEx (personal experiences of health and illness). J Nurs Manag. 2008:16(4):433-9.

61. Breuning M, Lucius-Hoene G, Burbaum C, Himmel W, Bengel J. Patient experiences and patient centeredness : the website project DIPEx Germany. Bundesgesundheitsbl Gesundheitsforsch Gesundheitsschutz. 2017;60(4):453-61.

62. Ziebland S, Lavie-Ajayi M, Lucius-Hoene G. The role of the internet for people with chronic pain: examples from the DIPEx international project. Br J Pain. 2015;9(1):62-4.

63. Smith SD, Farrugia LL, Harris V, Lee A, Carter SR, Blaszczynski A, et al. Evaluation of the influence of family and friends, and the internet on patient perceptions of long-term topical corticosteroid use. J Dermatolog Treat. 2017;28(7):642-6.

64. World Health Organisation. eHealth. https://www.who.int/ehealth/en/ Accessed 15 Apr 2019.

65. Greis C, Meier Zurcher C, Djamei V, Moser A, Lautenschlager S, Navarini AA. Unmet digital health service needs in dermatology patients. J Dermatolog Treat. 2018;29(7):643-7.

66. Ariens LF, Schussler-Raymakers FM, Frima C, Flinterman A, Hamminga E, Arents BW, et al. Barriers and facilitators to eHealth use in daily practice: perspectives of patients and professionals in dermatology. J Med Internet Res. 2017:19(9):e300.

67. van Os-Medendorp H, Koffijberg H, Eland-de Kok PC, van der Zalm A, de Bruin-Weller MS, Pasmans SG, et al. E-health in caring for patients with atopic dermatitis: a randomized controlled cost-effectiveness study of internet-guided monitoring and online self-management training. Br J Dermatol. 2012;166(5):1060-8.

68. Kornmehl H, Singh S, Johnson MA, Armstrong AW. Direct-access online Care for the Management of atopic dermatitis: a randomized clinical trial examining patient quality of life. Telemed J E Health. 2017;23(9):726-32.

69. Armstrong AW, Johnson MA, Lin S, Maverakis E, Fazel N, Liu FT. Patientcentered, direct-access online care for management of atopic dermatitis: a randomized clinical trial. JAMA Dermatol. 2015:151(2):154-60

70. Hoogenbosch B, Postma J, de Man-van Ginkel JM, Tiemessen NA, van Delden $\mathrm{J}$, van Os-Medendorp H. Use and the users of a patient portal: cross-sectional study. J Med Internet Res. 2018;20(9):e262.

71. Tongdee E, Markowitz O. Mobile app rankings in dermatology. Cutis. 2018; 102(4):252-6.

72. Ikoma A, Ebata T, Chantalat L, Takemura K, Mizzi F, Poncet M, et al. Measurement of nocturnal scratching in patients with pruritus using a Smartwatch: initial clinical studies with the itch tracker app. Acta Derm Venereol. 2018;99(3):268-73.

73. Irizarry T, DeVito DA, Curran CR. Patient portals and patient engagement: a state of the science review. J Med Internet Res. 2015;17(6):e148.

74. Kruse CS, Argueta DA, Lopez L, Nair A. Patient and provider attitudes toward the use of patient portals for the management of chronic disease: a systematic review. J Med Internet Res. 2015;17(2):e40.

75. UMC Utrecht. Eczeem [Eczema]. https://www.umcutrecht.nl/nl/Ziekenhuis/ Ziekte/Eczeem. Accessed 15 Apr 2019.

76. Foundation for Atopic Dermatitis. Research and Education. https://www. fondation-dermatite-atopique.org/en. Accessed 15 April 2019

77. American College of Allergy, Asthma \& Immunology. Controlling your Eczema (Atopic Dermatitis). https://acaai.org/controlling-your-eczemaatopic-dermatitis. Accessed 15 April 2019

78. Sauder MB, McEvoy A, Ramien ML. Prescribing success: developing an integrated prescription and eczema action plan for atopic dermatitis. J Am Acad Dermatol. 2016;75(6):1281-3. 
79. Shi WY, Nanda S, Lee K, Armstrong AW, Lio PA. Improving patient education with an eczema action plan: a randomized controlled trial. JAMA Dermatol. 2013;149(4):481-3.

80. Powell K, Le Roux E, Banks JP, Ridd MJ. Developing a written action plan for children with eczema: a qualitative study. Br J Gen Pract. 2018;68(667):e81-9.

81. American Academy of Dermatology. Eczema Action Plan. https://www.aad org/public/diseases/eczema/eczema-resource-center/controlling-eczema/ eczema-action-plan. Accessed 15 Apr 2019.

\section{Publisher's Note}

Springer Nature remains neutral with regard to jurisdictional claims in published maps and institutional affiliations.

Ready to submit your research? Choose BMC and benefit from:

- fast, convenient online submission

- thorough peer review by experienced researchers in your field

- rapid publication on acceptance

- support for research data, including large and complex data types

- gold Open Access which fosters wider collaboration and increased citations

- maximum visibility for your research: over $100 \mathrm{M}$ website views per year

At $\mathrm{BMC}$, research is always in progress.

Learn more biomedcentral.com/submissions 